\title{
Detection of antiskyrmions by topological Hall effect in Heusler compounds
}

\author{
Vivek Kumar $\odot,{ }^{1}$ Nitesh Kumar, ${ }^{1}$ Manfred Reehuis, ${ }^{2}$ Jacob Gayles, ${ }^{1}$ A. S. Sukhanov, ${ }^{1,3}$ Andreas Hoser, ${ }^{2}$ Françoise Damay, ${ }^{4}$ \\ Chandra Shekhar, ${ }^{1}$ Peter Adler, ${ }^{1}$ and Claudia Felser ${ }^{1, *}$ \\ ${ }^{1}$ Max Planck Institute for Chemical Physics of Solids, 01187 Dresden, Germany \\ ${ }^{2}$ Helmholtz-Zentrum Berlin für Materialien und Energie, 14109 Berlin, Germany \\ ${ }^{3}$ Institut für Festkörper- und Materialphysik, Technische Universität Dresden, 01069 Dresden, Germany \\ ${ }^{4}$ Laboratoire Léon Brillouin, CEA-CNRS, CEA Saclay, 91191 Gif-sur-Yvette, France
}

(Received 6 February 2019; revised manuscript received 23 October 2019; published 15 January 2020)

\begin{abstract}
Heusler compounds having $D_{2 d}$ crystal symmetry gained much attention recently due to the stabilization of a vortexlike spin texture called antiskyrmions in thin lamellae of $\mathrm{Mn}_{1.4} \mathrm{Pt}_{0.9} \mathrm{Pd}_{0.1} \mathrm{Sn}$ as reported in the work of Nayak et al. [Nature (London) 548, 561 (2017)]. Here we show that bulk $\mathrm{Mn}_{1.4} \mathrm{Pt}_{0.9} \mathrm{Pd}_{0.1} \mathrm{Sn}$ undergoes a spinreorientation transition from a collinear ferromagnetic to a noncollinear configuration of Mn moments below $135 \mathrm{~K}$, which is accompanied by the emergence of a topological Hall effect. We tune the topological Hall effect in $\mathrm{Pd}$ and $\mathrm{Rh}$ substituted $\mathrm{Mn}_{1.4} \mathrm{PtSn}$ Heusler compounds by changing the intrinsic magnetic properties and spin textures. A unique feature of the present system is the observation of a zero-field topological Hall resistivity with a sign change which indicates the robust formation of antiskyrmions.
\end{abstract}

DOI: 10.1103/PhysRevB.101.014424

\section{INTRODUCTION}

Topological magnetic textures show promise in many areas of technology, such as racetrack memory $[1,2]$ and neuromorphic computing $[3,4]$, due to the unique transport properties that allow for efficient manipulation $[5,6]$ and detection $[7,8]$ of the magnetic textures. This is a consequence of the realspace Berry curvature of the topological texture that can be tuned by the electronic structure $[9,10]$. One such topological texture is the skyrmion [11,12] which is found in chiral magnets [13] with a unit integer topological charge. Recently antiskyrmions (aSKs) [14], the antiparticles of the skyrmions, were stabilized in thin lamellae of the tetragonal inverse Heusler compound $\mathrm{Mn}_{1.4} \mathrm{Pt}_{0.9} \mathrm{Pd}_{0.1} \mathrm{Sn}$ for a broader field range and even above room temperature [15]. In this compound $\left(D_{2 d}\right.$ symmetry class), the Dzyaloshinskii-Moriya interaction (DMI) is anisotropic where the DM vectors along the $x$ and $y$ directions in the basal plane are of opposite sign and hence cause the stability of the aSKs [16]. The in-plane winding of the spin texture for an aSK is anisotropic with an opposite topological charge as compared to that of the azimuthally symmetric Bloch and Néel skyrmion of the same charge [17]. The topological winding induces a real-space Berry curvature that strongly influences all transport properties [18]. The topological Hall effect (THE) is one such electrical transport property that has been intrinsically linked to the skyrmions [8].

\footnotetext{
*claudia.felser@cpfs.mpg.de
}

Published by the American Physical Society under the terms of the Creative Commons Attribution 4.0 International license. Further distribution of this work must maintain attribution to the author(s) and the published article's title, journal citation, and DOI.
The THE results in a further component of the total Hall signal, in addition to the normal Hall and the magnetizationscaled anomalous Hall components [19]. It arises due to the emergent magnetic field which is a result of the finite Berryphase contributions from either real or momentum spaces when the conduction electrons interact with a noncoplanar (NCP) spin texture of finite chirality [20]. The THE was widely studied in the skyrmions hosting B20 compounds $[7,8,19,21-24]$. It was also observed in other systems possessing NCP spin structures, e.g., pyrochlore lattices [25,26], antiferromagnets $[27,28]$, spin glasses [29,30], and correlated systems such as oxide thin films [31,32].

A chemical substitution in place of the heavy element $\mathrm{Pt}$ in the noncentrosymmetric Heusler compound $\mathrm{Mn}_{1.4} \mathrm{PtSn}$ can allow for systematic control of the intrinsic properties that give rise to topological textures, namely spin-orbit coupling (SOC), electron occupation, and magnetization. These properties determine fundamental parameters such as the exchange interactions, the DMI, and the magnetocrystalline anisotropy (MCA). In turn, the competition of these parameters determines which magnetic texture is formed, i.e., a long-wavelength spiral or a topological spin texture. The presence of more than one magnetic sublattice and the competition between parallel and antiparallel exchange interactions in the noncentrosymmetric crystal can lead to a spin-

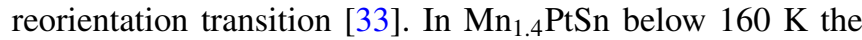
formation of a noncollinear spin structure was reported [15]. In this paper, we investigate the detailed magnetic structures of $\mathrm{Mn}_{1.4} \mathrm{Pt}_{0.9} \mathrm{Pd}_{0.1} \mathrm{Sn}$ below and above the spin-reorientation transition temperature $T_{\mathrm{SR}}$ and demonstrate that below $T_{\mathrm{SR}}$ a THE emerges with features which are evidence for the presence of aSKs in the bulk material. Fractional substitution of $\mathrm{Pt}$ by $\mathrm{Pd}$ (isoelectronic substitution) in $\mathrm{Mn}_{1.4} \mathrm{PtSn}$ is used to exclusively change the SOC, whereas substitution by $\mathrm{Rh}$ varies the electron occupancy of the bands and in consequence 
changes the SOC as well as the magnetization. Our results demonstrate the tunability of topological spin textures in the Heusler system which paves the way for systematic design of aSK phase containing compounds suitable for applications in spintronics. The THE can be used as a sensitive probe for characterizing the spin textures.

\section{EXPERIMENT}

Polycrystalline ingots of $\mathrm{Mn}_{1.4} \mathrm{Pt}_{1-x} \mathrm{Pd}_{x} \mathrm{Sn} \quad(x=0$, $0.1 \ldots 0.3)$ and $\mathrm{Mn}_{1.4} \mathrm{Pt}_{1-y} \mathrm{Rh}_{y} \mathrm{Sn}(y=0.1,0.2 \ldots 0.8)$ with the stoichiometric amounts of constituent elements were prepared by arc melting in the presence of $\mathrm{Ar}$ atmosphere. The synthesized ingots were sealed in an evacuated quartz tube and annealed at $873 \mathrm{~K}$ (Pd-substituted samples) and $1073 \mathrm{~K}$ (parent compound $\mathrm{Mn}_{1.4} \mathrm{PtSn}$ and Rh-substituted samples) for $7 \mathrm{~d}$ followed by quenching into an ice-water mixture. The phase purity and the crystal structure of the samples were examined by room-temperature powder x-ray diffraction using a Huber G670 camera [Guinier technique, $\lambda=1.54056 \AA\left(\mathrm{Cu}-\mathrm{K} \alpha_{1}\right.$ radiation $\left.)\right]$.

Neutron-powder-diffraction experiments of $\mathrm{Mn}_{1.4} \mathrm{Pt}_{0.9} \mathrm{Pd}_{0.1} \mathrm{Sn}$ were carried out on the instrument E6 at the BER II reactor of the Helmholtz-Zentrum Berlin, where powder-diffraction patterns were recorded between the diffraction angles $(2 \theta) 5.5^{\circ}$ and $136.5^{\circ}$. This instrument uses a pyrolytic graphite $(\mathrm{PG})$ monochromator selecting the neutron wavelength $\lambda=2.43 \AA$. For the experiment the powder sample was filled in a vanadium container (cylindrical) of diameter $6 \mathrm{~mm}$ and height $4 \mathrm{~cm}$. The temperature dependence of the crystal and magnetic structure was investigated between 2.4 and $449 \mathrm{~K}$ using an Orange Cryofurnace (AS Scientific Products Ltd., Abingdon, UK). In order to follow in detail the structural and magnetic changes, we collected in this temperature range 47 powder-diffraction patterns. Datasets at 2.4, 203, and $449 \mathrm{~K}$, were measured with an increased counting rate in order to determine the positional parameters and magnetic moments with good accuracy.

Neutron-powder-diffraction measurements of $\mathrm{Mn}_{1.4} \mathrm{Pt}_{1-y} \mathrm{Rh}_{y} \mathrm{Sn}(y=0.4,0.6,0.7$, and 0.8$)$ were taken on the cold-neutron two-axis powder diffractometer (instrument G 4-1) at Laboratoire Léon Brillouin, France. The neutron wavelength $\lambda=2.43 \AA$ was selected using a PG monochromator. Powdered samples were filled in a vanadium container (cylindrical) of diameter $6 \mathrm{~mm}$ and height $4 \mathrm{~cm}$. Samples were cooled down in a cryostat to $1.5 \mathrm{~K}$ and measurements were taken while warming in the $2 \theta$ range between $2^{\circ}$ and $82^{\circ}$. Rietveld refinements of all the powder-diffraction data were carried out with the program FULLPROF [34], using the nuclear scattering lengths $b(\mathrm{Mn})=-3.73 \mathrm{fm}, b(\mathrm{Pd})=5.91 \mathrm{fm}, b(\mathrm{Pt})=9.63 \mathrm{fm}$, and $b(\mathrm{Sn})=6.228 \mathrm{fm} \mathrm{[35]}$. The magnetic form factor of the Mn atoms was taken from Ref. [36].

Magnetization measurements were performed using a vibrating sample magnetometer (MPMS3, Quantum Design, $\mu_{0} H_{\max }=7 \mathrm{~T}$ ). The electrical transport properties were investigated using a physical property measurement system (PPMS9, Quantum Design, $\mu_{0} H_{\max }=9 \mathrm{~T}$ ). The samples were cut into rectangular-bar-shaped pieces with dimensions of approximately $3.5 \times 1.5 \times 0.5 \mathrm{~mm}^{3}$. Point contacts were made on the samples with $25-\mu$ m-diameter Pt wires and silver paint. The five-probe geometry was used for the Hall experiments. The Hall resistivity was measured at different temperatures between 2 and $300 \mathrm{~K}$ in the field range from -5 to $5 \mathrm{~T}$ in hysteresis mode by applying the field perpendicular to the rectangular surface of the bar. In order to avoid any artifact due to the demagnetization effect, both magnetization and resistivity measurements were performed on the same piece (length $x$ breadth $\times$ height) where the magnetic field was applied perpendicular to the rectangular surface (length $\times$ breadth) under identical conditions. It should be noted that in a superconducting coil trapped magnetic flux may lead to deviations of reported and real magnetic field. Especially, when measuring the magnetization, magnetoresistance, or the Hall resistivity of a soft ferromagnet in a field sweep through zero, the reversal of the hysteresis (i.e., of the coercive fields) may be observed [37]. The presented data were measured in an MPMS3 magnetometer with a 7-T magnet and a PPMS with a 9-T magnet, respectively. In field sweeps both systems typically produce a maximum field error of 20 Oe around zero field [37].

\section{RESULTS}

\section{A. Crystal structure and lattice parameters}

We determined the crystal structure and lattice parameters of the prepared samples by Rietveld refinement of the powder X-ray-diffraction patterns shown in Fig. S1 of Supplemental Material [38] using the FULLPROF program. The tetragonal crystal structure is retained in both the substitution series $\mathrm{Mn}_{1.4} \mathrm{Pt}_{1-x} \mathrm{Pd}_{x} \mathrm{Sn}$ and $\mathrm{Mn}_{1.4} \mathrm{Pt}_{1-y} \mathrm{Rh}_{y} \mathrm{Sn}$. All samples crystallize in the inverse tetragonal Heusler structure (space group $I \overline{4} 2 d$, No. 122). The lattice constants, $c / a$ ratios, and the cell volumes of $\mathrm{Mn}_{1.4} \mathrm{Pt}_{1-x} \mathrm{Pd}_{x} \mathrm{Sn}$ and $\mathrm{Mn}_{1.4} \mathrm{Pt}_{1-y} \mathrm{Rh}_{y} \mathrm{Sn}$ are shown in Fig. 1. The lattice constant $a$ increases continuously

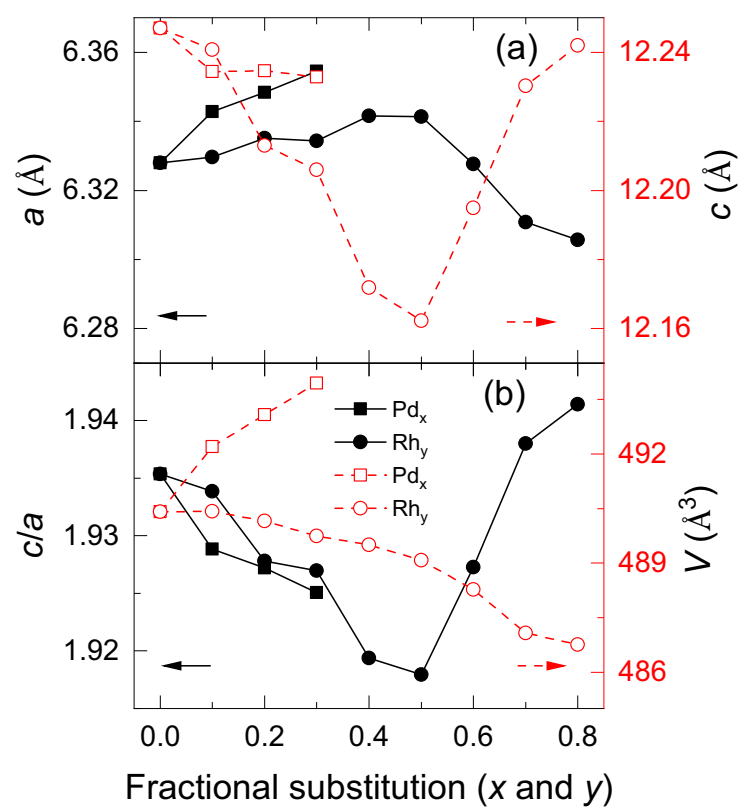

FIG. 1. Lattice constants (a and c), $c / a$ ratio and cell volume $V$ of $\mathrm{Mn}_{1.4} \mathrm{Pt}_{1-x} \mathrm{Pd}_{x} \mathrm{Sn}$ and $\mathrm{Mn}_{1.4} \mathrm{Pt}_{1-y} \mathrm{Rh}_{y} \mathrm{Sn}$. The size of the error bars is less than the symbol size. 

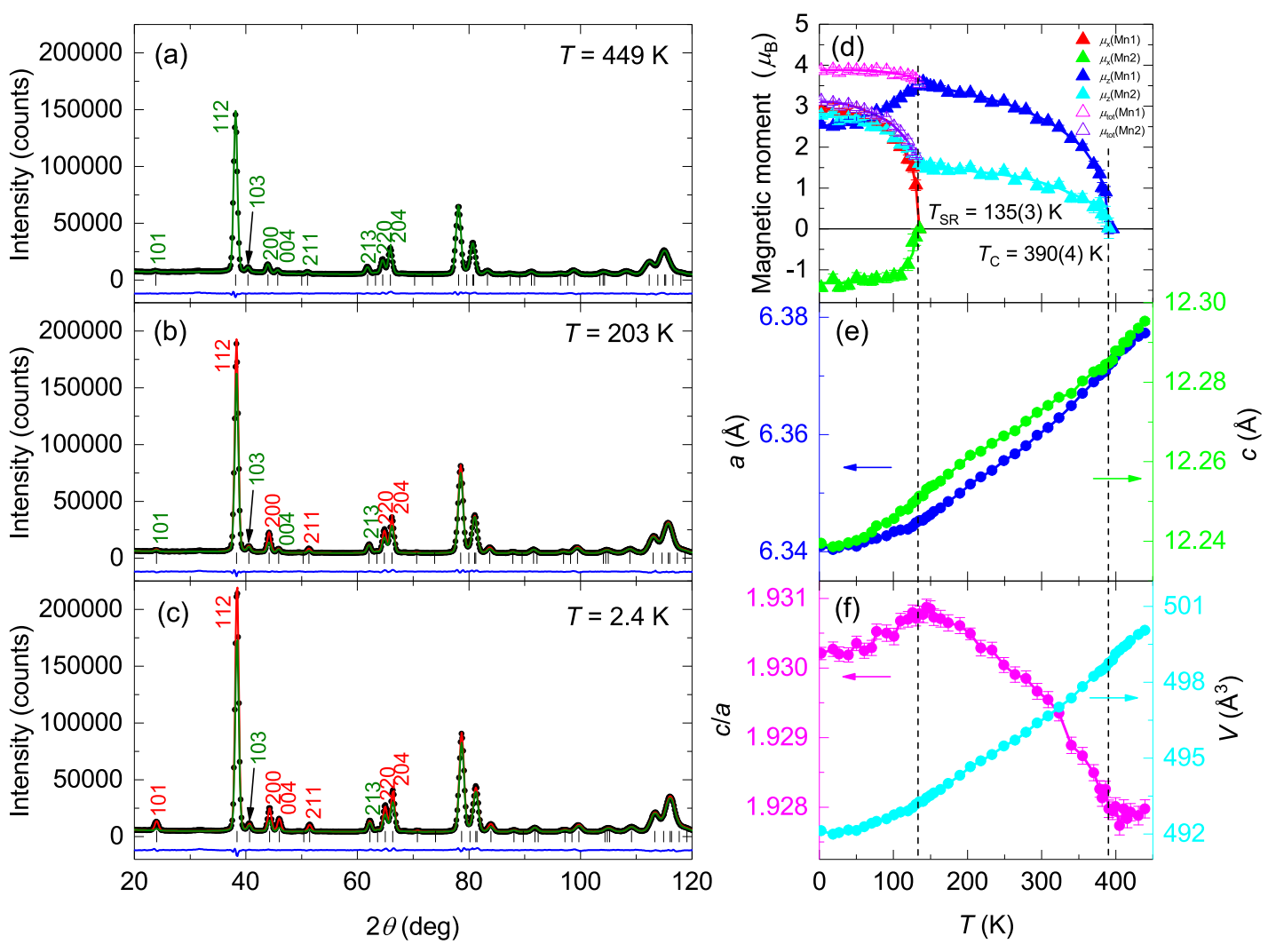

FIG. 2. Results of the Rietveld refinements of the neutron-powder-diffraction data of $\mathrm{Mn}_{1.4} \mathrm{Pt}_{0.9} \mathrm{Pd}_{0.1} \mathrm{Sn}$ collected with $\lambda=2.43 \AA$ at (a) 449 , (b) 203 , and (c) $2.4 \mathrm{~K}$, respectively. The crystal structure was refined in the tetragonal space group $I \overline{4} 2 d$. At $203 \mathrm{~K}$ the strongest magnetic intensity appears at the positions of the reflections 112, 200, 211, 220, and 204 (marked in red), indicating a ferromagnetic spin alignment of the $\mathrm{Mn}$ atoms. At $2.4 \mathrm{~K}$ the reflections 101 and 004 show additional magnetic intensities indicating a spin-reorientation transition. The calculated patterns (crystal structure in green, crystal and magnetic structure in red) are compared with the observed ones (black circles). The difference patterns (blue) as well as the positions (black bars) of the Bragg reflections are shown. In (d), (e), and (f) the temperature dependence of the total magnetic moment $\mu_{\text {tot }}$ and the magnetic moment components $\mu_{x}$ and $\mu_{z}$ of the Mn atoms, the lattice parameters $a$ and $c$, and the $c / a$ ratio and the unit-cell volume $V$ in $\mathrm{Mn}_{1.4} \mathrm{Pt}_{0.9} \mathrm{Pd}_{0.1} \mathrm{Sn}$ are depicted, respectively. The size of the error bars of $a, c$, and $V$ is less than the symbol size. Ferromagnetic ordering sets in at the Curie temperature $T_{\mathrm{C}}=390(4) \mathrm{K}$. Below $T_{\mathrm{SR}}=135(3) \mathrm{K}$ the $x$ components of the $\mathrm{Mn} 1$ and Mn2 moments show a ferrimagnetic spin alignment. The dotted vertical lines are a guide for the eye.

by Pd substitution whereas $c$ gets reduced slightly compared to the parent compound and remains almost constant with increasing $x$, thereby the $c / a$ ratio decreases and the cell volume increases. On the other hand, Rh substitution first increases $a$ and decreases $c$ up to $y=0.5$ and thereafter a reverse trend is observed, i.e., $a$ decreases and $c$ increases from $y=0.6$ to 0.8 . Consequently, the $c / a$ ratio decreases up to $y=0.5$ and then increases considerably. However, the cell volume decreases linearly up to $y=0.5$ and thereafter the decrement is nonmonotonic.

In this system, the tetragonal structure results from two cubic unit cells slightly displaced along the $c$ axis which leads to a $c / a$ ratio close to 2 . Hence, the degree of tetragonality increases when the $c / a$ ratio tends away from the value 2 , or in other words the $c / a$ ratio moves away from the pseudocubic condition $\left(c=a^{\prime}\right.$, where $\left.a^{\prime}=2 a\right)$. The decrease in the $c / a$ ratio for all $\mathrm{Pd}$ substitutions and moderate $\mathrm{Rh}$ substitutions up to $y=0.5$ suggests an enhanced tetragonality. Accordingly, higher Rh substitution, $y>0.5$, leads to a decrease in tetragonality as reflected in the drastic rise in the $c / a$ ratio.

\section{B. Neutron powder diffraction}

We investigated the detailed magnetic structure of the present system using neutron-powder-diffraction experiments. In Fig. 2 the neutron-powder-diffraction patterns of $\mathrm{Mn}_{1.4} \mathrm{Pt}_{0.9} \mathrm{Pd}_{0.1} \mathrm{Sn}$ are shown; the corresponding Rietveld refinement results are summarized in Table S1 of Supplemental Material [38]. The data collected at $449 \mathrm{~K}$ (well above the Curie temperature, $T_{\mathrm{C}}=390 \mathrm{~K}$ ) were successfully refined in the space group $I \overline{4} 2 d$ (No. 122) as shown in Fig. 2(a). In this space group two different Mn atoms (labeled as Mn1 and $\mathrm{Mn} 2)$ are located at the Wyckoff positions $8 d(x, 1 / 4,1 / 8)$ with $x \sim 0.73$ and $4 a(0,0,0)$ [in our case we used the second site generated by $(0,1 / 2,1 / 4)=(1 / 2,1 / 2,1 / 2)+(1 / 2,0,3 / 4)]$, while the $\mathrm{Sn}$ atoms are located at $8 d(x, 1 / 4,1 / 8)$ with $x \sim 0.28$. The refinements showed that the Mn2 atoms at the site $4 a$ only reach an occupancy of $0.772(19)$. For the Pt and $\mathrm{Pd}$ atoms, which are statistically distributed at the position $8 c(0,0, z)$ [in our case we used the second site generated by $\left.(0,1 / 2, z)=(1 / 2,1 / 2,1 / 2)+\left(1 / 2,0, z^{\prime}+3 / 4\right)\right]$ (with $z \sim 0.02)$, one obtains the occupancies $\operatorname{occ}(\mathrm{Pt})=0.902(22)$ 
and $\operatorname{occ}(\mathrm{Pd})=0.098(22)$. This finally gives the formula $\mathrm{Mn}_{1.39(1)} \mathrm{Pt}_{0.90(2)} \mathrm{Pd}_{0.10(2)}$ Sn. Alternatively, the crystal structure of $\mathrm{Mn}_{1.4} \mathrm{Pt}_{0.9} \mathrm{Pd}_{0.1} \mathrm{Sn}$ has been described earlier within space group $I \overline{4} 2 m$ (No. 121) [15]. In this space group additional reflections $(002,110,114,222,006$, etc.) are generated which are not observed in the experimental patterns. Accordingly, $\mathrm{Mn}_{1.4} \mathrm{Pt}_{0.9} \mathrm{Pd}_{0.1} \mathrm{Sn}$ crystallizes in the space group $I \overline{4} 2 d$.

A previous report [15] and our magnetization measurements (see Supplemental Material [38]) indicated the presence of a transition into a ferro- or ferrimagnetic state at the Curie temperature $T_{\mathrm{C}} \sim 390 \mathrm{~K}$ followed by a spin reorientation transition, which sets in at $T_{\mathrm{SR}}<150 \mathrm{~K}$. Therefore, in order to determine the high-temperature magnetic structure of $\mathrm{Mn}_{1.4} \mathrm{Pt}_{0.9} \mathrm{Pd}_{0.1} \mathrm{Sn}$, we used the dataset collected at $203 \mathrm{~K}$ well between the two transition temperatures $T_{\mathrm{C}}$ and $T_{\mathrm{SR}}$. The strongest magnetic intensities are superimposed on the positions of the nuclear reflections 112, 200, 220, and 204 [shown in Fig. 2(b)], indicating a ferromagnetic ordering of the $\mathrm{Mn}$ atoms with a propagation vector $\boldsymbol{k}=0$. Due to the fact that magnetic intensity is observable on the reflections 200 and 220 it can be assumed that the magnetic moments are predominantly aligned parallel to the $c$ axis. Therefore, we used a magnetic structure model where only the $z$ components of the magnetic moments $\mu_{z}(\mathrm{Mn} 1)$ and $\mu_{z}(\mathrm{Mn} 2)$ were allowed to vary; see Table S1 in Supplemental Material [38]. The magnetic moments were found to be $\mu_{z}(\mathrm{Mn} 1)=3.32(3) \mu_{B}$ and $\mu_{z}(\mathrm{Mn} 2)=1.70(6) \mu_{B}$. The excellent agreement between the observed and calculated intensity of the reflection 004 suggests that no additional ferromagnetic component occurs in the $a b$ plane at $203 \mathrm{~K}$. The neutron powder-diffraction analysis reveals that the high-temperature magnetic configuration has a collinear ferromagnetic spin alignment along the tetragonal axis, in contrast to the previous suggestion of a ferrimagnetic spin structure [15]. The proposed magnetic structure was based on the general model of $\mathrm{Mn}_{2}$-based inverse Heusler compounds, which frequently have a ferrimagnetic configuration due to antiparallel spin alignment between $\mathrm{Mn}$ atoms at different sites $[39,40]$. A simulation of a ferrimagnetic model demonstrates that strong magnetic Bragg intensity is generated at the position of the relatively weak nuclear reflection 101 which is not observed.

Below $135 \mathrm{~K}$, our neutron-powder-diffraction data showed a spontaneous increase of magnetic intensity on the reflections 101 and 004. The strong increase of the magnetic intensity of the 004 reflection indicates the presence of an additional spin alignment in the $a b$ plane. We focused on the dataset collected at $2.4 \mathrm{~K}$ [shown in Fig. 2(c)] to solve the groundstate magnetic configuration. The refinements showed that it is impossible to determine the moment direction within the $a b$ plane. Therefore, we have set the moment direction of $\mathrm{Mn} 1$ and Mn2 parallel to the $a$ axis. The moments of the two $\mathrm{Mn}$ atoms are coupled antiparallel resulting in a ferrimagnetic ordering in the $a b$ plane. At $2.4 \mathrm{~K}$ the magnetic moments reach in the plane the moment values $\mu_{x}(\mathrm{Mn} 1)=2.89(7) \mu_{B}$ and $\mu_{x}(\mathrm{Mn} 2)=-1.65(11) \mu_{B}$. The observed magnetic structure is compatible with the symmetry of the space group $I \overline{4} 2 d$ as shown in Supplemental Material [38].

It is interesting to see that from 203 to $2.4 \mathrm{~K}$ the $z$ component of $\mathrm{Mn} 1$ is strongly decreasing [from 3.32(3) to

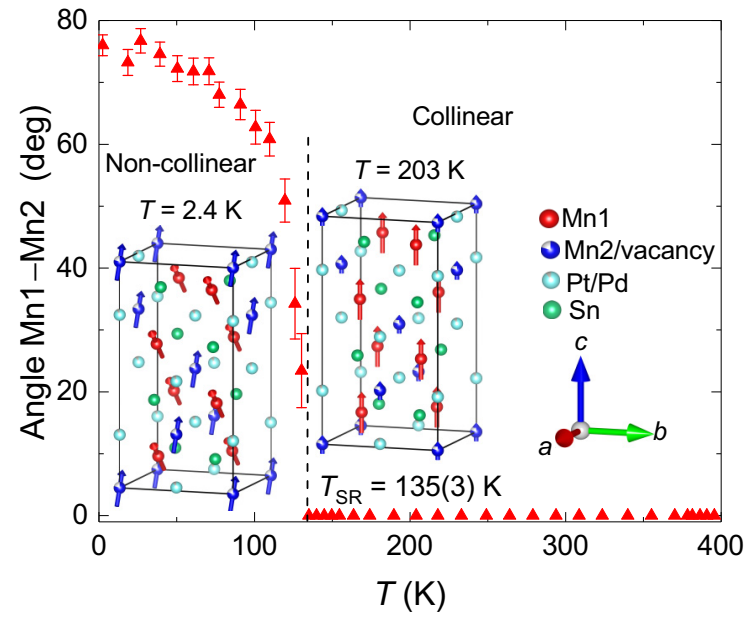

FIG. 3. Magnetic structure of $\mathrm{Mn}_{1.4} \mathrm{Pt}_{0.9} \mathrm{Pd}_{0.1} \mathrm{Sn}$ at 2.4 and $203 \mathrm{~K}$, and the variation of the angle between Mn1 and Mn2 moments as a function of temperature.

2.56(6) $\mu_{B}$ ], whereas that of $\mathrm{Mn} 2$ is strongly increasing [from 1.70(6) to 3.13(7) $\mu_{B}$ ]. This shows that the moment of Mn1 is much stronger tilted from the $c$ axis $\left(48.5^{\circ}\right)$ than that of Mn2 $\left(27.7^{\circ}\right)$. The total moments of the two manganese atoms are $\mu_{\text {tot }}(\mathrm{Mn} 1)=3.87(5) \mu_{B}$ and $\mu_{\text {tot }}(\mathrm{Mn} 2)=3.54(5) \mu_{B}$.

From Fig. 2(d), where the temperature dependence of the magnetic moments is shown, it is apparent that the spin-reorientation transition at $T_{\mathrm{SR}}$ leads to a spontaneous increase of the total moments of the atoms Mn1 and Mn2. The total magnetic moments derived from the neutron data compare well with those from the magnetization measurements, see Supplemental Material [38]. From the temperature dependence of the moments the transition temperatures $T_{\mathrm{C}}=$ 390(4) $\mathrm{K}$ and $T_{\mathrm{SR}}=135(3) \mathrm{K}$ are obtained. In Figs. 2(e) and 2(f) we show the thermal variation of the lattice parameters $a$ and $c$, and the cell volume, $V$, which are continuously decreasing to $2.4 \mathrm{~K}$. Both magnetic transitions are reflected in slight anomalies, which is most apparent for the $c / a$ ratio [shown in Fig. 2(f)].

Our neutron investigations demonstrate that $\mathrm{Mn}_{1.4} \mathrm{Pt}_{0.9} \mathrm{Pd}_{0.1} \mathrm{Sn}$ displays a collinear ferromagnetic spin alignment along the tetragonal axis in the temperature range $T_{\mathrm{SR}}<T<T_{\mathrm{C}}$, whereas the magnetic structure transforms into a noncollinear spin configuration for $T<T_{\mathrm{SR}}$. In Fig. 3 the magnetic structures below $(2.4 \mathrm{~K})$ and above $(200 \mathrm{~K}) T_{\mathrm{SR}}$, as well as the angle between the Mn1 and Mn2 moments are shown. The spin-reorientation transition involves spin canting of each Mn sublattice as well as an increase in the total moments. These findings are consistent with the temperature-dependent magnetization data presented in Fig. S3 of Supplemental Material [38]. The low-temperature spin structure is noncollinear, but still coplanar. Recently, a noncoplanar spin arrangement has been discussed as a candidate structure for $\mathrm{Mn}_{1.4} \mathrm{PtSn}$ even at zero field, where an additional antiferromagnetic component may be present parallel to $y$ [41]. This order should generate magnetic intensity at the position of the reflection 002. From our data of $\mathrm{Mn}_{1.4} \mathrm{Pt}_{0.9} \mathrm{Pd}_{0.1} \mathrm{Sn}$ collected at $2.4 \mathrm{~K}$ we were not able to find significant magnetic intensity at this position. 


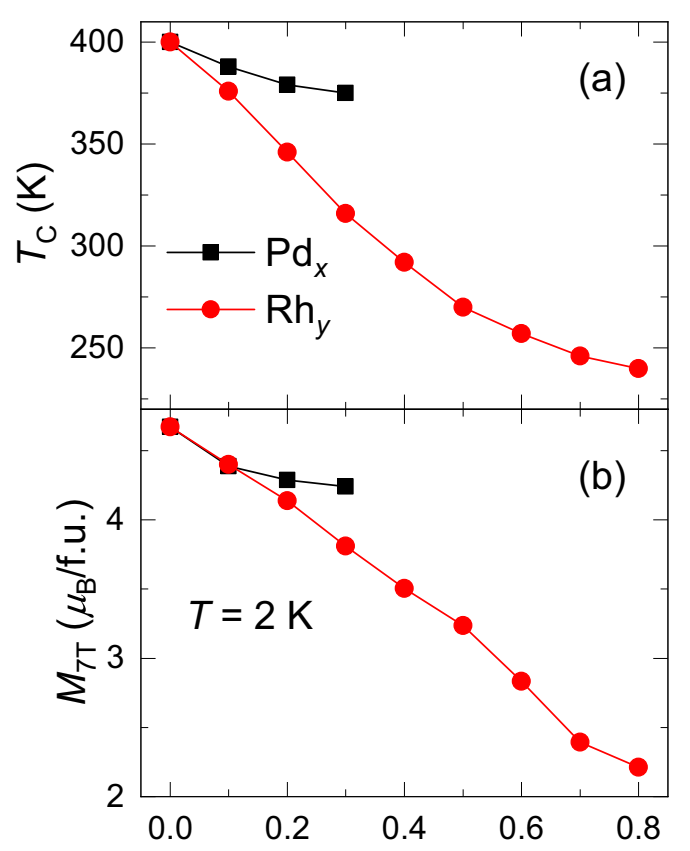

Fractional substitution ( $x$ and $y$ )

FIG. 4. (a) Curie temperature and (b) magnetization at $7 \mathrm{~T}$ and $2 \mathrm{~K}$ of $\mathrm{Mn}_{1.4} \mathrm{Pt}_{1-x} \mathrm{Pd}_{x} \mathrm{Sn}$ and $\mathrm{Mn}_{1.4} \mathrm{Pt}_{1-y} \mathrm{Rh}_{y} \mathrm{Sn}$.

Simulations showed that any magnetic moment parallel to $y$ is expected to be smaller than $0.2 \mu_{B}$.

Neutron patterns of $\mathrm{Mn}_{1.4} \mathrm{Pt}_{0.6} \mathrm{Rh}_{0.4} \mathrm{Sn}$ verify that for moderate degrees of $\mathrm{Rh}$ substitution a noncollinear spin structure and collinear ferromagnetic structure are retained below and above $T_{\mathrm{SR}}$, respectively, whereas for higher substitution levels $y>0.5$ an essentially collinear ferrimagnetic (FiM) spin configuration with both Mn moments lying in the basal plane emerges at all temperatures below $T_{\mathrm{C}}$ (see Supplemental Material [38]). The change in spin structure is associated with a decrease in the tetragonal distortion of the crystal structure for $y>0.5$ as discussed in Sec. III A. (cf. Fig. 1). The neutrondiffraction data, however, cannot exclude the persistence of a small spin canting which might lead to a topological Hall effect in an external magnetic field.

\section{Magnetic properties}

The evolution of the magnetic properties for both substitution series is depicted in Fig. 4 and Figs. S4 and S5 of Supplemental Material [38]. The parent sample exhibits a $T_{\mathrm{C}}$ of $\sim 400 \mathrm{~K}$ which reduces to $375 \mathrm{~K}$ by increasing the $\mathrm{Pd}$ substitution to $x=0.3$. On the other hand, Rh substitution results in a $T_{\mathrm{C}}$ of $246 \mathrm{~K}$ for $y=0.8$. The magnetization does not saturate even at the maximum applied field of $7 \mathrm{~T}$. The parent compound $\mathrm{Mn}_{1.4} \mathrm{PtSn}$ bears a moment of $4.6 \mu_{B} /$ f.u. at $7 \mathrm{~T}$ at $2 \mathrm{~K}$. For both $\mathrm{Pd}$ and $\mathrm{Rh}$ substitution, the magnetization at $7 \mathrm{~T}$ and $2 \mathrm{~K}$ decreases with increasing degree of substitution $x$ or $y$. However, the variation is rather small in the case of $\mathrm{Pd}(x)$ as compared to $\mathrm{Rh}(y)$ substitution even for the same amount of substitution. For instance, the magnetizations at $7 \mathrm{~T}$ approach 4.24 and $3.85 \mu_{B} /$ f.u. for $x=0.3$ and $y=$ 0.3 , respectively. An approximately linear decrease of the
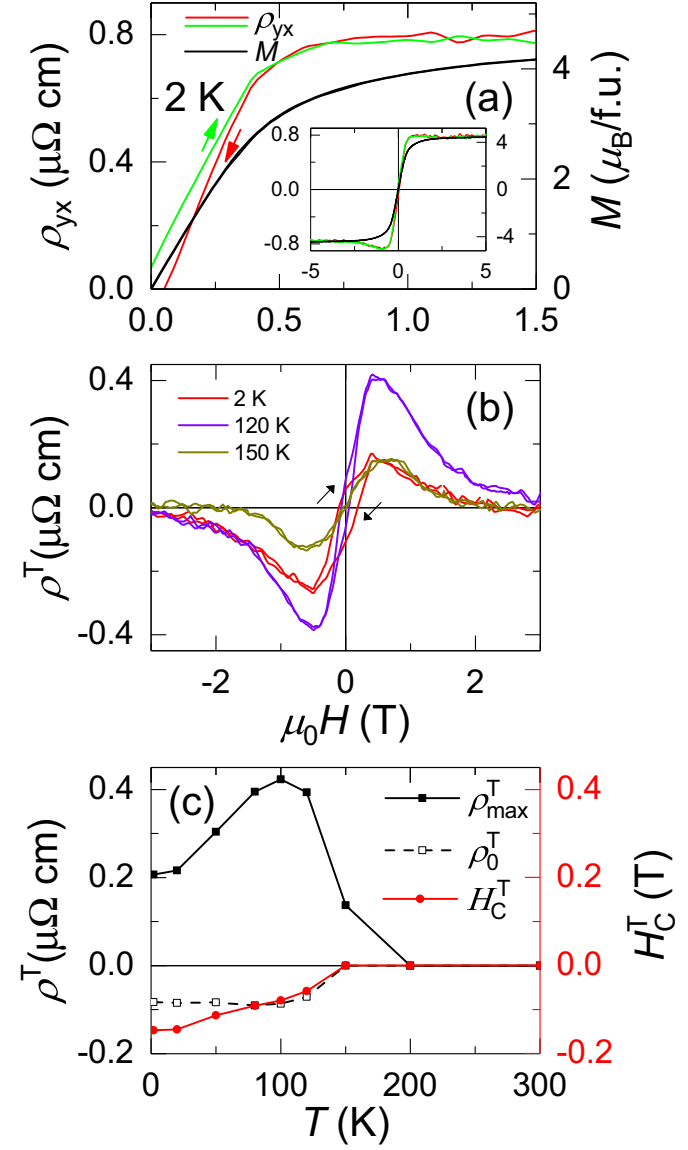

FIG. 5. (a) Hall resistivity (left axis) and magnetization (right axis) of $\mathrm{Mn}_{1.4} \mathrm{Pt}_{0.9} \mathrm{Pd}_{0.1} \mathrm{Sn}$ between 0 and $1.5 \mathrm{~T}$. Decreasing and increasing field-sweep directions in $\rho_{y x}$ (red and green lines, respectively) are indicated by arrows. The inset shows full hysteresis loops between -5 and $+5 \mathrm{~T}$. (b) Topological Hall resistivity at 2, 120, and $150 \mathrm{~K}$. (c) Temperature dependence of maximum and zero-field value and coercivity of the topological Hall resistivity.

magnetization is observed with increasing $y$, i.e., decreasing number of valence electrons, for Rh contents up to $y=0.5$, which demonstrates a Slater-Pauling-like reduction [42].

\section{Topological Hall effect}

In Fig. 5(a) we show the total Hall resistivity together with the magnetization as a function of an external field for $\mathrm{Mn}_{1.4} \mathrm{Pt}_{0.9} \mathrm{Pd}_{0.1} \mathrm{Sn}$. Two distinctions in the Hall resistivity $\rho_{y x}(H)$ as compared to the magnetization $M(H)$ are observed: (1) an enhanced feature just below the field, above which the magnetization tends to saturate, and (2) a large coercivity $(\sim-450 \mathrm{Oe})$ in the Hall resistivity hysteresis (within $\pm 0.5 \mathrm{~T})$ which is the reverse of that of the magnetic isotherm. As mentioned in the Experiment section the maximum error in the magnetic field in our superconducting magnets is 20 Oe; such small field errors cannot be responsible for the hysteresis loop reversals observed for the present material. Indeed, the observation of an enhanced feature below the saturation field in $\rho_{y x}(H)$ indicates that actually a noncoplanar spin configuration emerges from the canted spins below $T_{\mathrm{SR}}$ which is induced by application of a magnetic field in the Hall 
(a)
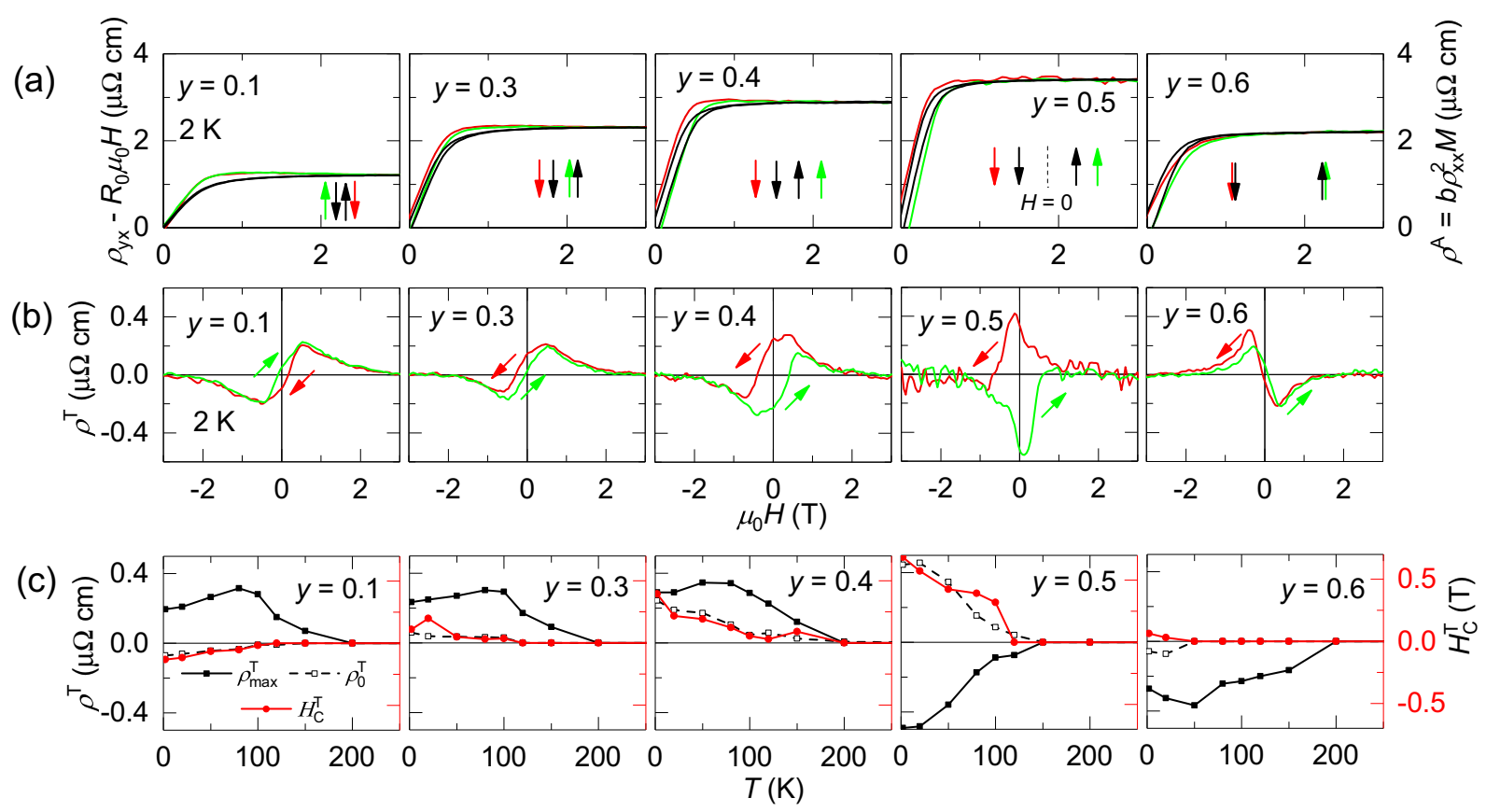

FIG. 6. (a) Corrected Hall resistivity $\rho_{y x}-R_{0} \mu_{0} H$ (left axis, red and green lines) and the calculated anomalous Hall resistivity $\rho^{\mathrm{A}}\left(=b \rho_{x x}^{2} M\right)$ (right axis, black lines) at $2 \mathrm{~K}$ of $\mathrm{Mn}_{1.4} \mathrm{Pt}_{1-y} \mathrm{Rh}_{y} \mathrm{Sn}$. The black arrows detail the magnetization hysteresis around zero fields with the down (up) arrow for the decreasing (increasing) field sweep. The red (green) arrow shows the position of the Hall resistivity for decreasing (increasing) field sweep around zero fields with respect to the magnetization. (b) Topological Hall resistivity at $2 \mathrm{~K}$ and (c) the temperature-dependent parameters characterizing $\rho^{\mathrm{T}}$.

experiment and gives rise to a THE. On the other hand, the inverse hysteretic observation at lower field in $\rho_{y x}(H)$ suggests the presence of aSKs whose cores are antiparallel to the field direction and which are stabilized by the MCA. In B20 compounds this effect is observed when the anisotropy is induced by the thin-film limit $[7,22,43]$, while in the compounds with $D_{2 d}$ symmetry the anisotropy is inherent to the crystal lattice. In addition, microstructural defects like twins and grain boundaries of the polycrystalline samples may also act as pinning centers to stabilize the aSKs. Previous reports on analogous Heusler compounds, e.g., bulk $\mathrm{Mn}_{2} \mathrm{PtSn}$ [44] and thin films of $\mathrm{Mn}_{2} \mathrm{PtSn}$ [45], $\mathrm{Mn}_{2-x} \mathrm{PtSn}$ [46], and $\mathrm{Mn}_{2} \mathrm{RhSn}$ [47] also revealed a THE but did not show an inverse hysteresis in the $\rho_{y x}$ loop. In Fig. 5(b) the topological Hall resistivity $\rho^{\mathrm{T}}$ at $T=2,120$, and $150 \mathrm{~K}$ is shown (the method for the extraction of $\rho^{\mathrm{T}}$ is shown in Supplemental Material [38]). $\rho^{\mathrm{T}}$ displays an inverse hysteresis as compared to the magnetization at 2 and $120 \mathrm{~K}\left(<T_{\mathrm{SR}}\right)$, whereas no hysteresis is present at $150 \mathrm{~K}\left(>T_{\mathrm{SR}}\right)$. In Fig. 5(c) we plot three features of the THE as a function of temperature: $\rho_{\max }^{\mathrm{T}}$, the average of the maximum values with the sign determined by the positive field value; $\rho_{0}^{\mathrm{T}}=\left(\rho_{0-}^{\mathrm{T}}-\rho_{0+}^{\mathrm{T}}\right) / 2$, the zero-field value; and $H_{\mathrm{C}}^{\mathrm{T}}=\left(H_{\mathrm{C}}^{+}-H_{\mathrm{C}}^{-}\right) / 2$, the coercivity in the $\rho^{\mathrm{T}}$ loops. The $-(+)$ sign corresponds to a decreasing (increasing) field sweep. $\rho_{\max }^{\mathrm{T}}$ reaches a maximum of $0.42 \mu \Omega \mathrm{cm}$ at 100 $\mathrm{K}$ and decreases to zero above $T_{\mathrm{SR}}$. It is unclear whether the finite but small $\rho_{\max }^{\mathrm{T}}$ value at $150 \mathrm{~K}\left(T>T_{\mathrm{SR}}\right)$ in the collinear magnetic phase is an artifact or actually is of topological origin. However, at higher temperatures $\left(T \gg T_{\mathrm{SR}}\right) \rho_{\max }^{\mathrm{T}}$ is nearly zero (see Fig. S11 of Supplemental Material [38]). $H_{\mathrm{C}}^{\mathrm{T}}$ (red) continuously decreases with temperature, due to the fluctuation of the moments at higher temperatures. $\rho_{0}^{\mathrm{T}}$, most interestingly, is constant, $-0.08 \mu \Omega \mathrm{cm}$, up to $T_{\mathrm{SR}}$, a further indication of an aSK state, where $\rho^{\mathrm{T}}$ is expected to be independent of the variation in longitudinal resistivity [19]. Further evidence of aSKs at $H=0$ is obtained by the opposite signs of $\rho_{\max }^{\mathrm{T}}$ and $\rho_{0}^{\mathrm{T}}$, which can only be caused by a sign change in the topological texture on going from a NCP structure to an aSK phase. In the work of Nayak et al. [15], the aSK phase was even stable above $T_{\mathrm{SR}}$ in thin lamellae of $\mathrm{Mn}_{1.4} \mathrm{Pt}_{0.9} \mathrm{Pd}_{0.1} \mathrm{Sn}$, which is probably related to the reduced dimensionality. By contrast, the present study of Hall experiments demonstrates that bulk polycrystalline materials show aSKs only in the noncollinear magnetic phase $\left(<T_{\mathrm{SR}}\right)$.

Upon increasing the Pd content up to $x=0.3$, the largest possible Pd substitution level, only slight changes in magnetic and transport properties are observed (see Supplemental Material [38]). This implies that a moderate change in SOC due to variation of the Pd substitution does not change much the physical situation reflected by Fig. 5. By contrast, substitution of Rh for Pt has more pronounced implications for the properties. In particular, the magnetization features a strong Slater-Pauling-like reduction with decreasing electron count as well as a decrease in $T_{\mathrm{C}}$ (see Fig. 4). Analysis of the field dependence of the magnetization indicates that the MCA in the present system is strengthened by increasing the Rh content (see Fig. S6 of Supplemental Material [38]).

In Fig. 6 the Hall transport properties of $\mathrm{Mn}_{1.4} \mathrm{Pt}_{1-y} \mathrm{Rh}_{y} \mathrm{Sn}$ are summarized. In Fig. 6(a) we show the experimental Hall resistivity corrected for the normal Hall contribution $\rho_{y x}-R_{0} \mu_{0} H\left(=\rho^{\mathrm{A}}+\rho^{\mathrm{T}}\right)$ for decreasing and increasing field sweeps at $2 \mathrm{~K}$ for $0.1 \leqslant y \leqslant 0.6$. Here $R_{0}$ is the normal Hall coefficient. The calculated anomalous Hall resistivity 
TABLE I. Coercive fields (in Oe) of $\mathrm{Mn}_{1.4} \mathrm{Pt}_{1-y} \mathrm{Rh}_{y} \mathrm{Sn}$.

\begin{tabular}{lcrr}
\hline \hline$y$ & Coercivity of $M(H), H_{\mathrm{C}}^{\mathrm{M}}$ & Coercivity of $\rho_{y x}, H_{\mathrm{C}}^{\mathrm{H}}$ & Coercivity of $\rho^{\mathrm{T}}, H_{\mathrm{C}}^{\mathrm{T}}$ \\
\hline 0.1 & $96 \pm 3$ & $-169 \pm 31$ & $-1357 \pm 43$ \\
0.3 & $252 \pm 17$ & $285 \pm 77$ & $1105 \pm 21$ \\
0.4 & $418 \pm 16$ & $690 \pm 56$ & $4194 \pm 110$ \\
0.5 & $396 \pm 49$ & $1030 \pm 91$ & $5802 \pm 460$ \\
0.6 & $672 \pm 35$ & $769 \pm 66$ & $633 \pm 1$ \\
\hline \hline
\end{tabular}

$\rho^{\mathrm{A}}\left(=b \rho_{x x}^{2} M\right)$ is also shown. The compounds for $y<0.3$ display similar features in the Hall signals as observed for the $\mathrm{Mn}_{1.4} \mathrm{Pt}_{1-x} \mathrm{Pd}_{x} \mathrm{Sn}$ compounds. However, further increment in the $\mathrm{Rh}$ content results in a larger coercivity in the magnetization, $H_{\mathrm{C}}^{\mathrm{M}}$, and the Hall resistivity, $H_{\mathrm{C}}^{\mathrm{H}}$, follows the magnetization hysteresis with respect to the sweep directions. In addition, the magnitude of the coercivity of $\rho_{y x}$ differs significantly from $H_{\mathrm{C}}^{\mathrm{M}}$ up to $y=0.5$, whereas it is nearly equal for $y \geqslant 0.6$ (see Table I). This is further shown as a schematic of arrows in Fig. 6(a). The increase in the magnetization coercivity $\left(H_{\mathrm{C}}^{\mathrm{M}}\right)$ with increase in the Rh content is consistent with the increasing MCA and anisotropy field estimated from the law of approach to saturation (see Fig. S6 [38]).

In Figs. 6(b) and 6(c) we show the $\rho^{\mathrm{T}}$ curves at $2 \mathrm{~K}$ and the temperature dependence of the topological Hall parameters ( $\rho^{\mathrm{T}}$ and $H_{\mathrm{C}}^{\mathrm{T}}$ values), respectively. A sign change from negative to positive in $H_{\mathrm{C}}^{\mathrm{T}}$ and consequently also in $\rho_{0}^{\mathrm{T}}$ occurs at $y=0.3$. For $y=0.5$, the topological parameters reach a maximum. As indicated by the neutron-diffraction studies, up to $y=0.5$ a noncollinear spin structure below $T_{\mathrm{SR}}$ is retained, with a nearly constant angle between the two Mn sublattices of $\sim 80^{\circ}$. However, for $y \geqslant 0.6$, the Mn sublattices approach the collinear FiM state in the whole temperature range and the topological Hall parameters sharply decrease.

In Fig. 7 the results of the topological $\left(H_{\mathrm{C}}^{\mathrm{T}}\right)$ and magnetic $\left(H_{\mathrm{C}}^{\mathrm{M}}\right)$ coercive field are presented together with $\rho_{\max }^{\mathrm{T}}$ and $\rho_{0}^{\mathrm{T}}$ at $2 \mathrm{~K}$. The dependence of the parameters on the Rh content can be related to the presence of three distinct magnetic structures. For Rh contents $0.6 \leqslant y \leqslant 0.8$ (yellow background) the spins align antiferromagnetically with a larger moment on the Mn1 site in a FiM configuration. Here, $H_{\mathrm{C}}^{\mathrm{T}}$ is significantly low owing to the similar coercivity in $\rho_{y x}(H)$ and $M(H)$ curves. Nevertheless, we found a finite negative $\rho_{\max }^{\mathrm{T}}$ and $\rho_{0}^{\mathrm{T}}$ which may be due to the small canting of the antiferromagnetic order vector or it can be an error generated in the extraction of the topological Hall resistivity (see Supplemental Material [38]). Both $\rho_{\max }^{\mathrm{T}}$ and $\rho_{0}^{\mathrm{T}}$ monotonously decrease to zero as the $\mathrm{Rh}$ content reaches $y=0.8$.

In the range of $0.25<y<0.6$ (green background), the spins form a NCP spin structure induced by the magnetic field. The variation of the MCA and the DMI with the SOC (coupling constant $\lambda$ ) and magnetization $(M)$ can be expressed by the relations $K_{\text {ani }} \sim \lambda^{2}$ [48] and $D \sim \lambda M^{2}$ [49], where $K_{\text {ani }}$ and $D$ are the anisotropy and DMI constant, respectively. Moreover, their strengths largely depend on the electronic states nearby the Fermi level which are expected to be varied to a large extent by Rh substitution (changing the number of valence electrons). A lower magnetization in this composition range may impede the formation of an aSK state as a result of overcompensation of the DMI by the MCA. Both $\rho_{\max }^{\mathrm{T}}$ and
$\rho_{0}^{\mathrm{T}}$ are additionally maximized in this region, however with an opposite sign at the maximum $(y=0.5)$. This is due to the change in the electronic structure, as also evidenced in the normal Hall effect, where the slope of $\rho_{y x}$ changes sign near $y=0.5$ (see Fig. S13 [38]).

Finally, the aSK phase (blue background) occurs in both $\mathrm{Rh}$ and Pd substituted samples in the composition ranges $0<y<0.25$ and $0 \leqslant x \leqslant 0.3$, respectively. The aSK phase is stabilized at lower fields $\left(\mu_{0} H \leqslant|0.5| \mathrm{T}\right)$ and remained stable at zero field. The $\rho_{\max }^{\mathrm{T}}$ has a nearly constant value of $\sim 0.2 \mu \Omega \mathrm{cm}$. Furthermore, $H_{\mathrm{C}}^{\mathrm{M}}$ is smallest in this range but its sign is also opposite to that of $H_{\mathrm{C}}^{\mathrm{T}}$. This is a key feature of the formation of aSKs. In this composition range, due to the enhanced magnetization, the DMI is sufficiently large to induce transformation of the NCP structure to the aSK phase, whereas the MCA is primarily important for the survival of the aSK at zero field. The opposite sign for $\rho_{0}^{\mathrm{T}}$ and $\rho_{\max }^{\mathrm{T}}$ is due to the change in topology of the magnetic spin structure.

The $\rho_{\max }^{\mathrm{T}}$ found in the present system is due to the NCP spin structure, which displays the largest value of $\sim 0.5 \mu \Omega \mathrm{cm}$, and is comparable to that observed in other similar Heusler compounds as bulk $\mathrm{Mn}_{2} \mathrm{PtSn}(1.53 \mu \Omega \mathrm{cm})$ [44], thin films of $\mathrm{Mn}_{2} \mathrm{PtSn}(0.5 \mu \Omega \mathrm{cm})$ [45], and $\mathrm{Mn}_{2-x} \mathrm{PtSn}(1.2 \mu \Omega$ $\mathrm{cm}$ ) [46]. However, these cases did not show the reversal of

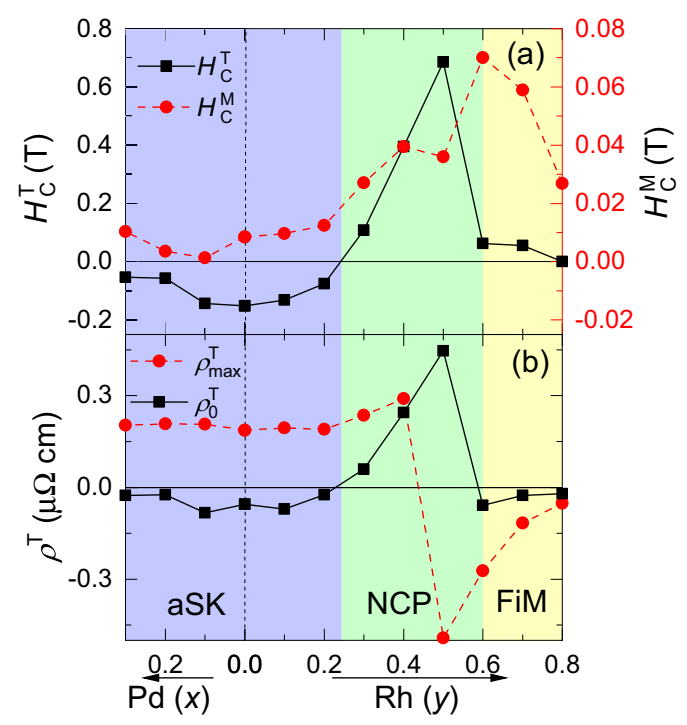

FIG. 7. (a) Coercive field of topological Hall resistivity (left axis) and magnetization (right axis) and (b) topological Hall resistivity of $\mathrm{Mn}_{1.4} \mathrm{Pt}_{1-x} \mathrm{Pd}_{x} \mathrm{Sn}$ and $\mathrm{Mn}_{1.4} \mathrm{Pt}_{1-y} \mathrm{Rh}_{y} \mathrm{Sn}$ at $2 \mathrm{~K}$. The compounds are classified in three composition ranges corresponding to the aSK phase, NCP spin structure, and FiM configuration which are indicated by blue, green, and yellow colors. 
hysteresis in the Hall resistivity compared to that of the magnetization, which is attributed to the aSK phase. The presence of aSKs results in a finite value of $\rho_{0}^{\mathrm{T}}$ with a negative sign, which within the present series of compounds has the largest magnitude of $\sim 0.08 \mu \Omega \mathrm{cm}$ for $x=0.1$. The THE in the B20 compounds due to the stabilization of skyrmions has been well described by the size of their spin texture. The emergent magnetic field $\left(B_{\mathrm{em}}\right)$ shows quadratic decrement with the size $\left(L_{\mathrm{sk}}\right)$ of the skyrmion in the adiabatic limit, $B_{\mathrm{em}} \propto 1 / L_{\mathrm{sk}}^{2}$ [18]. For instance, MnGe [19] and FeGe [22] display $\rho^{\mathrm{T}}$ values of 0.16 and $0.08 \mu \Omega \mathrm{cm}$ at a finite field for $L_{\mathrm{sk}}=3$ and $70 \mathrm{~nm}$, respectively. Intriguingly, despite the comparatively large size of the aSKs in $\mathrm{Mn}_{1.4} \mathrm{Pt}_{0.9} \mathrm{Pd}_{0.1} \mathrm{Sn}(\sim 150 \mathrm{~nm})$ [15], we find a large topological Hall resistivity even at zero fields. This is likely due to the role of the electronic structure in the topological Hall constant $R^{\mathrm{T}}=\rho^{\mathrm{T}} / B_{\text {em }} \quad$ [24], and provides the possibility to engineer even a larger THE in aSK phases with smaller periods.

\section{CONCLUSIONS}

We have shown the THE due to the robust formation of antiskyrmions below $T_{\mathrm{SR}}$ in the bulk $\mathrm{Mn}_{1.4} \mathrm{PtSn}$ system with fractional substitution of $\mathrm{Pt}$ by $\mathrm{Pd}$ and $\mathrm{Rh}$. The competition between the DMI and the MCA is crucial for the transformation of a noncoplanar spin structure to the antiskyrmion phase. Furthermore, we expect the antiskyrmion phase to be stable for wider composition and temperature ranges in thin plate samples, as indicated by our previous work (Ref. [15]). This leads to the possibility of having smaller-sized antiskyrmions, which can also be achieved by substitution of Pt by another heavy metal like Ir. Lastly, the ferrimagnetic configurations found for large $\mathrm{Rh}$ substitutions open the door to antiferromagnetic spintronics based on aSKs. Particularly useful would be ferrimagnetic materials tuned to the compensation point as compensated ferrimagnetic aSKs would travel in a straight-line trajectory and yet would be easily detectable due to different electronic properties of the constituent elements [54].

\section{ACKNOWLEDGMENTS}

The authors acknowledge funding by the Deutsche Forschungsgemeinschaft (DFG, German Research Foundation) under SPP 2137 (Project No. 403502666) and EU FET Open RIA Grant No. 766566 "ASPIN". We would like to thank Ajaya K. Nayak and Walter Schnelle for discussion.
[1] S. Parkin and S.-H. Yang, Nat. Nanotechnol. 10, 195 (2015).

[2] A. Fert, V. Cros, and J. Sampaio, Nat. Nanotechnol. 8, 152 (2013).

[3] D. Pinna, F. A. Araujo, J.-V. Kim, V. Cros, D. Querlioz, P. Bessiere, J. Droulez, and J. Grollier, Phys. Rev. Appl. 9, 064018 (2018).

[4] Z. He and D. Fan, arXiv:1705.02995v1.

[5] X. Z. Yu, N. Kanazawa, W. Z. Zhang, T. Nagai, T. Hara, K. Kimoto, Y. Matsui, Y. Onose, and Y. Tokura, Nat. Commun. 3, 988 (2012).

[6] F. Jonietz, S. Mühlbauer, C. Pfleiderer, A. Neubauer, W. Münzer, A. Bauer, T. Adams, R. Georgii, P. Böni, R. A. Duine, K. Everschor, M. Garst, and A. Rosch, Science 330, 1648 (2010).

[7] J. C. Gallagher, K. Y. Meng, J. T. Brangham, H. L. Wang, B. D. Esser, D. W. McComb, and F. Y. Yang, Phys. Rev. Lett. 118, 027201 (2017).

[8] A. Neubauer, C. Pfleiderer, B. Binz, A. Rosch, R. Ritz, P. G. Niklowitz, and P. Böni, Phys. Rev. Lett. 102, 186602 (2009).

[9] D. Xiao, M.-C. Chang, and Q. Niu, Rev. Mod. Phys. 82, 1959 (2010).

[10] K. Manna, Y. Sun, L. Muechler, J. Kübler, and C. Felser, Nat. Mater. Rev. 3, 244 (2018).

[11] A. N. Bogdanov and D. A. Yablonskiǐ, Zh. Eksp. Teor. Fiz. 95, 178 (1989) [Sov. Phys. JETP 68, 101 (1989)].

[12] U. K. Rößler, A. N. Bogdanov, and C. Pfleiderer, Nature (London) 442, 797 (2006).

[13] S. Mühlbauer, B. Binz, F. Jonietz, C. Pfleiderer, A. Rosch, A. Neubauer, R. Georgii, and P. Böni, Science 323, 915 (2009).

[14] A. N. Bogdanov, U. K. Rößler, M. Wolf, and K.-H. Müller, Phys. Rev. B 66, 214410 (2002).

[15] A. K. Nayak, V. Kumar, T. Ma, P. Werner, E. Pippel, R. Sahoo, F. Damay, U. K. Rößler, C. Felser, and S. S. P. Parkin, Nature (London) 548, 561 (2017).
[16] S. Huang, C. Zhou, G. Chen, H. Shen, A. K. Schmid, K. Liu, and Y. Wu, Phys. Rev. B 96, 144412 (2017).

[17] W. Koshibae and N. Nagaosa, Nat. Commun. 7, 10542 (2016).

[18] N. Nagaosa and Y. Tokura, Nat. Nanotechnol. 8, 899 (2013).

[19] N. Kanazawa, Y. Onose, T. Arima, D. Okuyama, K. Ohoyama, S. Wakimoto, K. Kakurai, S. Ishiwata, and Y. Tokura, Phys. Rev. Lett. 106, 156603 (2011).

[20] M. Onoda, G. Tatara, and N. Nagaosa, J. Phys. Soc. Jpn. 73, 2624 (2004).

[21] T. Yokouchi, N. Kanazawa, A. Tsukazaki, Y. Kozuka, M. Kawasaki, M. Ichikawa, F. Kagawa, and Y. Tokura, Phys. Rev. B 89, 064416 (2014).

[22] S. X. Huang and C. L. Chien, Phys. Rev. Lett. 108, 267201 (2012).

[23] Y. Li, N. Kanazawa, X. Z. Yu, A. Tsukazaki, M. Kawasaki, M Ichikawa, X. F. Jin, F. Kagawa, and Y. Tokura, Phys. Rev. Lett. 110, 117202 (2013).

[24] C. Franz, F. Freimuth, A. Bauer, R. Ritz, C. Schnarr, C. Duvinage, T. Adams, S. Blügel, A. Rosch, Y. Mokrousov, and C. Pfleiderer, Phys. Rev. Lett. 112, 186601 (2014).

[25] Y. T. Y. Taguchi, Y. Oohara, H. Yoshizawa, N. Nagaosa, and Y. Tokura, Science 291, 2573 (2001).

[26] Y. Machida, S. Nakatsuji, Y. Maeno, T. Tayama, T. Sakakibara, and S. Onoda, Phys. Rev. Lett. 98, 057203 (2007).

[27] C. Sürgers, G. Fischer, P. Winkel, and H. V. Löhneysen, Nat. Commun. 5, 3400 (2014).

[28] B. G. Ueland, C. F. Miclea, Y. Kato, A. Valenzuela, R. D. McDonald, R. Okazaki, P. H. Tobash, M. A. Torrez, F. Ronnig, R. Movshovich, Z. Fisk, E. D. Bauer, I. Martin, and J. D. Thompson, Nat. Commun. 3, 1067 (2012).

[29] F. W. Fabris, P. Pureur, J. Schaf, V. N. Vieira, and I. A. Campbell, Phys. Rev. B 74, 214201 (2006). 
[30] T. Taniguchi, K. Yamanaka, H. Sumioka, T. Yamazaki, Y. Tabata, and S. Kawarazaki, Phys. Rev. Lett. 93, 246605 (2004).

[31] Y. Ohuchi, Y. Kozuka, M. Uchida, K. Ueno, A. Tsukazaki, and M. Kawasaki, Phys. Rev. B 91, 245115 (2015).

[32] L. Vistoli, W. Wang, A. Sander, Q. Zhu, B. Casals, R. Cichelero, A. Barthélémy, S. Fusil, G. Herranz, S. Valencia, R. Abrudan, E. Weschke, K. Nakazawa, H. Kohno, J. Santamaria, W. Wu, V. Garcia, and M. Bibes, Nat. Phys. 15, 67 (2019).

[33] O. Meshcheriakova, S. Chadov, A. K. Nayak, U. K. Rößler, J. Kübler, G. André, A. A. Tsirlin, J. Kiss, S. Hausdorf, A. Kalache, W. Schnelle, M. Nicklas, and C. Felser, Phys. Rev. Lett. 113, 087203 (2014).

[34] J. Rodríguez-Carvajal, Physica B 192, 55 (1993).

[35] V. F. Sears, International Tables for Crystallography, Vol. C (Kluwer Academic, Dordrecht, 1995), p. C 383.

[36] P. J. Brown, International Tables for Crystallography, Vol. C (Kluwer Academic, Dordrecht, 1995), p. C 391.

[37] G. Mastrogiacomo, J. F. Löffler, and N. R. Dilley, Appl. Phys. Lett. 92, 082501 (2008); Application Note 1070-207, Using PPMS Superconducting Magnets at Low Fields, Quantum Design, San Diego (2009); Application Note 1500-021, Correcting for the Absolute Field Error Using the Pd Standard, San Diego (2017).

[38] See Supplemental Material at http://link.aps.org/supplemental/ 10.1103/PhysRevB.101.014424 for further details about data evaluation and additional structural, neutron, magnetic characterization, and transport data, which includes Refs. [50-53].

[39] L. Wollmann, S. Chadov, J. Kübler, and C. Felser, Phys. Rev. B 90, 214420 (2014).

[40] L. Wollmann, S. Chadov, J. Kübler, and C. Felser, Phys. Rev. B 92, 064417 (2015).

[41] P. Vir, J. Gayles, A. S. Sukhanov, N. Kumar, F. Damay, Y. Sun, J. Kübler, C. Shekhar, and C. Felser, Phys. Rev. B 99, 140406(R) (2019).
[42] S. Chadov, J. Kiss, and C. Felser, Adv. Funct. Mater. 23, 832 (2013).

[43] N. Kanazawa, M. Kubota, A. Tsukazaki, Y. Kozuka, K. S. Takahashi, M. Kawasaki, M. Ichikawa, F. Kagawa, and Y. Tokura, Phys. Rev. B 91, 041122(R) (2015).

[44] Z. H. Liu, A. Burigu, Y. J. Zhang, H. M. Jafri, X. Q. Ma, E. K. Liu, W. H. Wang, and G. H. Wu, Scr. Mater. 143, 122 (2018).

[45] Y. Li, B. Ding, X. Wang, H. Zhang, W. Wang, and Z. Liu, Appl. Phys. Lett. 113, 062406 (2018).

[46] P. Swekis, A. Markou, D. Kriegner, J. Gayles, R. Schlitz, W. Schnelle, S. T. B. Goennenwein, and C. Felser, Phys. Rev. Mater. 3, 013001(R) (2019).

[47] K. G. Rana, O. Meshcheriakova, J. Kübler, B. Ernst, J. Karel, R. Hillebrand, E. Pippel, P. Werner, A. K. Nayak, C. Felser, and S. S. P. Parkin, New J. Phys. 18, 085007 (2016).

[48] P. Bruno, Phys. Rev. B 39, 865 (1989).

[49] F. Freimuth, R. Bamler, Y. Mokrousov, and A. Rosch, Phys. Rev. B 88, 214409 (2013).

[50] Y. Tokunaga, X. Z. Yu, J. S. White, H. M. Rønnow, D. Morikawa, Y. Taguchi, and Y. Tokura, Nat. Commun. 6, 7638 (2015).

[51] K. Karube, J. S. White, N. Reynolds, J. L. Gavilano, H. Oike, A. Kikkawa, F. Kagawa, Y. Tokunaga, H. M. Rønnow, Y. Tokura, and Y. Taguchi, Nat. Mater. 15, 1237 (2016).

[52] S. V. Andreev, M. I. Bartashevich, V. I. Pushkarskya, V. N. Maltsev, L. A. Pamyatnykh, E. N. Tarasov, N. V. Kudrevatykh, and T. Goto, J. Alloys Compd. 260, 196 (1997).

[53] Y. Huh, P. Kharel, A. Nelson, V. R. Shah, J. Pereiro, P. Manchanda, A. Kashyap, R. Skomski, and D. J. Sellmyer, J. Phys.: Condens. Matter 27, 076002 (2015).

[54] L. Caretta, M. Mann, F. Büttner, K. Ueda, B. Pfau, C. M. Günther, P. Hessing, A. Churikova, C. Klose, M. Schneider, D. Engel, C. Marcus, D. Bono, K. Bagschik, S. Eisebitt, and G. S. D. Beach, Nat. Nanotechnol. 13, 1154 (2018). 